\title{
Phase-field Theory of Edges in an Anisotropic Crystal
}

\author{
By A. A. WheELER \\ School of Mathematics, University of Southampton, Highfield, Southampton, UK
}

In the presence of sufficiently strong surface energy anisotropy the equilibrium shape of an isothermal crystal may include corners or edges. Models of edges have, to date, involved the regularisation of the corresponding free boundary problem resulting in equilibrium shapes with smoothed out edges. In this paper we take a new approach and consider how a phase-field model, which provides a diffuse description of an interface, can be extended to the consideration of edges by an appropriate regularisation of the underlying mathematical model. Using the method of matched asymptotic expansions we develop an approximate solution which corresponds to a smoothed out edge from which we are able to determine the associated edge energy.

Keywords: Anisotropy, Interface, Phase-Field, Regularisation, Surface Energy

\section{Introduction}

The effect of anisotropic surface energy on determining the equilibrium shape of a solid crystal in contact with its own liquid phase is a classical problem in material science. The anisotropy of the surface energy of the interface is an expression of the underlying symmetry of the atomic lattice in the crystal. This, in turn, affects the macroscopic shape of the equilibrium interface. This shape is a circle (or sphere in three dimensions) in the absence of anisotropy. The presence of anisotropy changes the shape and, for strong anisotropy, it may be energetically favourable for the interface to exclude a range orientations (so called 'missing orientations') that correspond to higher surface energies, resulting in the formation of corners, edges or facets in the interface shape.

This situation has been studied for over a century, dating back to Gibbs (1878) and Curie (1885). The first solution was given by Wulff (1901) using the well known Wulff construction which employs the polar plot of the surface energy. The Wulff theory was reviewed and refined by Herring (1951) and subsequently further interpretations have been developed by Burton, Cabrera \& Frank (1951), Frank (1963), Hoffmann \& Cahn (1972) Cahn and Hoffmann (1974) and Andreev (1981). In two dimensions the surface energy, $\gamma(\theta)$, may be expressed as dependent on the polar angle of the normal vector to the interface, $\theta$. The equilibrium theory shows that interfaces includes corners when the so-called 'surface stiffness', $\gamma+\gamma_{\theta \theta}$, is negative.

The above developments are based on macroscopic models in which the interface is represented as a surface with no thickness, so-called 'sharp interface' models. The notion that an interface has an intrinsic thickness dates back to Lord Rayleigh (1892) and van der Waals (1893) in the context of an interface between two fluids. Since then this notion has been generalised to a wide range of interfaces and phase transitions, see Rowlinson and Widon (1989) for a comprehensive review. In the last 
two decades these ideas have been applied to solid-liquid interfaces using so-called phase-field models. These models introduce an order parameter, $\phi$, known as the phase-field. The phase-field takes a constant prescribed value in each bulk phase. Interfaces are represented as regions in which the value of the phase field changes between the bulk phase values. The contours of the phase field describe the shape and location of the solid-liquid interface. These models date back to Caginalp (1985, 1986) and Langer (1986). They can be considered as a mathematical device that allows a reformulation of the free-boundary problems associated with the sharp interface formulation. They have been successfully developed to describe a wide range of phase transitions, including eutectic and peritectic alloys Karma (1994), Wheeler et al (1996), Nestler \& Wheeler (1998). They have also been used to model numerically a wide range of microstructure including dendritic growth, Kobayashi (1992), Wheeler et al (1993), Karma \& Rappel (1997) and Ostwald ripening, Warren and Murray (1996).

Surface energy anisotropy was first included in phase-field models by Kobayashi (1992). Subsequently, Wheeler and McFadden $(1996,1997)$ made a connection with the Cahn-Hoffman $\xi$-vector which provides a powerful mechanism to understand anisotropic interfaces in the context of a phase-field model.

The dynamic evolution of an interface with anisotropic surface energy is problem that has been extensively studied. A particularly interesting situation is the evolution of a planar interface in a direction for which the surface stiffness is negative, and corresponds to a missing orientation. In this case the underlying mathematical problem is a backward parabolic partial differential equation, resulting in an illposed problem so that small wave length disturbances grow preferentially causing "blow up" of the solution on the smallest length scale. This occurs in both the free boundary formulation, Di Carlo et al (1992) and the phase-field model, Wheeler (1999).

To date, the focus has been on the free boundary formulation which involves applying a regularisation by including higher terms to the surface energy, see Stewart \& Goldenfeld (1992), Liu \& Metiu (1993), Golovin et al (1998, 1999, 2001, 2003). This may involve adding surface diffusion or evaporation or more simply allowing the surface energy to depend also on curvature so that $\gamma=\tilde{\gamma}(\theta)+\beta \mathcal{K}^{2}$, where $\mathcal{K}$ is the curvature of the interface and $\beta$ is a constant. This can be expected to prevent the formation of corners by making high curvature of the interface energetically unfavourable thus smoothing out the corners in the equilibrium shape and damping the growth of short wave length disturbances in the evolution of an interface. Recently, Spencer (2004) found asymptotic solutions that describe the shape of a smoothed corner in the equilibrium shape in the limit $\beta \rightarrow 0$.

In this paper we consider the regularization of the phase-field model to investigate the smoothing of corners. We confine our attention to two-dimensional interfaces and build on the anistropic phase-field model that employs the generalised $\xi$-vector of Wheeler and McFadden $(1996,1997)$. We show that in the limit of the coefficient of the regularising term going to zero a thin edge region is established within the interface which connects the two adjacent interfaces. Within the edge region the interface orientation varies smoothly through the range of missing orientations predicted by both the free-boundary and phase-field models in the absence of any regularisation. We use the method of matched asymptotic expansions to find an asymptotic solution in the five different adjacent regions comprising the two bulk

Article submitted to Royal Society 
phases, the two adjoining interfaces interfaces and the edge region. In the edge region itself we derive, at leading order, a nonlinear fourth order partial differential equation for the interface orientation. Despite the nonlinearity we are able reduce its solution to a single numerical quadrature. The edge problem has the character to a phase transition of an interface and is governed by a steady Allen-Cahn equation where the double-well nature of the associated potential is related to the form of $\gamma(\theta)$. This analogy of the edge to a phase transition at an interface was first made by Cabrera (1963) and later by Stewart and Goldenfeld (1992) in the context of the sharp interface model. We go on to define and evaluate the edge energy associated with the presence of the edge region. In addition, we show that there is an underlying stress tensor associated with the regularised phase-field equation, which is a natural extension of the stress tensor for the the phase-field model in the absence of regularisation, Wheeler and McFadden (1997).

In the next section we set out the basic elements of the Cahn Hoffman $\xi$-vector theory developed for the sharp interface model. We also describe the phase-field model and how it can be extended to the anisotropic case by the introduction of a generalised $\xi$-vector. In section 3 we formulate the regularisation of this model appropriate to the consideration of edges. In section 4 we employ the method of matched asymptotic expansions to analyse corners and compute the corner energy.

\section{Theory of Interfaces with Anisotropic Surface Energy}

(a) Sharp Interface Theory: the $\xi$-vector

Here we consider the isothermal equilibrium shape of a solid in contact with its liquid phase when the surface energy of the solid/liquid interface, $\gamma$, depends on the local orientation of the interface. We restrict our consideration to a cylindrical solid crystal. Specifically, the interface, $\mathcal{I}$, is considered to be a cylindrical surface whose cross-sectional shape is represented as a curve, $\mathcal{S}$, in the $(x, y)$-plane, see Figure 1. An edge in $\mathcal{I}$ is represented by a straight line parallel to the $z$-axis emanating from a corner in $\mathcal{S}$ at which its gradient is discontinuous.

In the simple case where the surface energy is isotropic (i.e., independent of local interface orientation) the equilibrium shape, $\mathcal{S}$, is a circle, whose radius $\hat{R}$ is related to the temperature of the system, $\hat{T}$, through the Gibbs-Thomson equation

$$
\hat{\gamma}_{0} \mathcal{K}=\Delta \hat{f}=\frac{\hat{L}\left(\hat{T}_{M}-\hat{T}\right)}{\hat{T}_{M}} .
$$

Here $\hat{\gamma}_{0}$ is the value of the surface energy, $\mathcal{K},=1 / \hat{R}$, is the curvature of the interface, $\Delta \hat{f}$ is the free energy density difference between the solid and liquid phases, $\hat{L}$ is the latent heat per unit volume, and $\hat{T}_{M}$ is the equilibrium melting temperature of the material. The Gibbs-Thomson equation results from minimising the total free energy of the system, comprising the contributions from the two bulk phases and the interface through its surface energy, subject to the constraint of constant volume of solid.

When the surface energy depends on the local orientation of the interface we represent it by

$$
\hat{\gamma}=\hat{\gamma}_{0} \Gamma(\vec{n})
$$


where $\Gamma(\vec{n})$ describes the dependence of the surface energy on the orientation of the interface through the vector $\vec{n}$, the outward unit normal to the solid/liquid interface. In this situation Cahn and Hoffman (1974) showed that the Gibbs-Thomson equation (2.1) becomes

$$
\nabla_{\mathcal{S}} \cdot \vec{\xi}=\frac{\hat{L}\left(\hat{T}-\hat{T}_{M}\right)}{\hat{T}_{M}} .
$$

Here $\vec{\xi}$ is the so-called Cahn-Hoffman $\xi$-vector and $\nabla_{\mathcal{S}}$. is the surface divergence operator on the interfacial surface $\mathcal{S}$. Taylor et al (1992) provided the following convenient definition of the $\xi$-vector: First extend the domain of the argument of $\Gamma$ from unit to general vectors by making a homogeneous degree one extension:

$$
\Gamma(\vec{p})=|\vec{p}| \Gamma\left(\frac{\vec{p}}{|\vec{p}|}\right),
$$

for all non-zero vectors $\vec{p}$. With this definition the Cahn-Hoffman $\xi$-vector is defined by

$$
\xi_{i}=\hat{\gamma}_{0} \frac{\partial \Gamma(\vec{p})}{\partial p_{i}},
$$

where subscript $i$ represents the $i$ 'th component of a vector. It follows from this definition that the $\xi$-vector has the properties

$$
\hat{\gamma}=\vec{\xi} \cdot \vec{n}, d \hat{\gamma}=\vec{\xi} \cdot d \vec{p}, 0=\vec{p} \cdot d \vec{\xi}
$$

The above definition (2.5) for $\vec{\xi}$ in fact holds more generally for fully three dimensional interfaces. However, in the cylindrical case, if we express the anisotropy through the dependence of $\gamma$ on the angle of the unit normal, $\vec{n}$, to (say) the $x$-axis, denoted by $\theta$, then $\vec{\xi}$ can be expressed as

$$
\vec{\xi}=\gamma(\theta) \vec{e}_{r}+\gamma_{\theta}(\theta) \vec{e}_{\theta}
$$

where $\vec{e}_{r}$ and $\vec{e}_{\theta}$ are the polar unit vectors associated with $\theta$. It follows that the modified Gibbs-Thomson equation (2.3) is

$$
\left[\hat{\gamma}+\hat{\gamma}_{\theta \theta}\right] \mathcal{K}=\frac{\hat{L}\left(\hat{T}-\hat{T}_{M}\right)}{\hat{T}_{M}} .
$$

There is geometrical interpretation of the $\xi$-vector which follows from its definition, given by equation (2.5), in which it is described as a vector perpendicular to the inverse $\gamma$ polar plot: $r=1 / \hat{\gamma}(\theta)$.

Equation (2.3) is satisfied by $\vec{x} \propto \vec{\xi}$ and thus the interfacial shape, $\mathcal{S}$, is given by $\vec{\xi}$ itself. Specifically, the $\xi$-vector plot, defined by the locus of $\vec{\xi}$ as a function of $\theta$, describes $\mathcal{S}$. Edges form in the interface shape when the inverse gamma plot becomes non-convex. This corresponds to $\hat{\gamma}+\hat{\gamma}_{\theta \theta}$ becoming negative for a range of interfacial orientations, $\theta$. In this situation the $\xi$-vector plot becomes multi-valued, developing "wings". This is shown in Figure 2 for the case $\Gamma(\theta)=1+0.2 \cos (\theta)$. The equilibrium shape comprises the inner envelope with the orientations corresponding to a wing absent resulting in a point of discontinuity of the gradient and hence a corner in $\mathcal{S}$. At the corner the $\xi$-vector (and hence $\mathcal{S}$ ) is nevertheless continuous. We 
will denote $\vec{\xi}$ at the corner by $\vec{\xi}$, it is determined by a common tangent construction on the inverse gamma plot.

For an isotropic surface energy the minimisation of the total energy associated with the interface is accomplished by reducing its overall length, which results in a surface tension force acting within the local tangent plane of the interface. The presence of surface energy anisotropy means that the minimisation of the surface energy of an interfacial element can be achieved by rotation as well as length contraction. Thus the surface tension force no longer remains confined locally to the tangent plane of the interface. Cahn and Hoffman showed that the surface tension force, $\overrightarrow{d F}$, acting on an interfacial element is given by

$$
d \vec{F}=\vec{\xi} \times d \vec{l}
$$

where $d \vec{l}$ represents a line element in $\mathcal{I}$. At an edge the balance of forces from the two adjoining portions of the interface results in

$$
\left(\vec{\xi}_{1}-\vec{\xi}_{2}\right) \times \vec{k}=0
$$

where $\vec{\xi}_{1}$ and $\vec{\xi}_{2}$ are the $\xi$-vectors of each interface evaluated at the corner and $\vec{k}$ is the tangent vector to the edge, being the unit vector in the $z$-direction. The continuity of $\vec{\xi}$ at an edge is an expression of the local force balance there. In Figure 3 we show the configuration of an edge. Using (2.7) we can express the common value of the $\xi$-vector, $\vec{\xi}^{\star}$, in terms of $\vec{\xi}$ at each of the two adjacent interfaces in terms of the basis unit vectors $\vec{N}$ and $\vec{M}$ shown in Figure 3. Thus

$$
\begin{aligned}
\vec{\xi}^{\star} & =\vec{\xi}_{1}=\left[\Gamma\left(\theta_{1}\right) \cos \left(\theta_{1}\right)-\sin \left(\theta_{1}\right) \Gamma^{\prime}\left(\theta_{1}\right)\right] \vec{N}+\left[\Gamma\left(\theta_{1}\right) \sin \left(\theta_{1}\right)+\cos \left(\theta_{1}\right) \Gamma^{\prime}\left(\theta_{1}\right)\right] \vec{M}(2.1 \\
\vec{\xi}^{\star} & =\vec{\xi}_{2}=\left[\Gamma\left(\theta_{2}\right) \cos \left(\theta_{2}\right)-\sin \left(\theta_{2}\right) \Gamma^{\prime}\left(\theta_{2}\right)\right] \vec{N}-\left[\Gamma\left(\theta_{2}\right) \sin \left(\theta_{2}\right)+\cos \left(\theta_{2}\right) \Gamma^{\prime}\left(\theta_{2}\right)\right] \vec{M}(.2
\end{aligned}
$$

Considering the orientation of $\vec{N}$ and $\vec{M}$ we have that $\vec{\xi}_{1} \cdot \vec{M}=\overrightarrow{\xi_{2}} \cdot \vec{M}=0$ which gives that

$$
\begin{aligned}
& \Gamma\left(\theta_{1}\right) \sin \left(\theta_{1}\right)+\cos \left(\theta_{1}\right) \Gamma^{\prime}\left(\theta_{1}\right)=0 \\
& \Gamma\left(\theta_{2}\right) \sin \left(\theta_{2}\right)+\cos \left(\theta_{2}\right) \Gamma^{\prime}\left(\theta_{2}\right)=0
\end{aligned}
$$

The force balance (2.10) may then be expressed as

$$
\Gamma\left(\theta_{1}\right) \cos \left(\theta_{1}\right)-\sin \left(\theta_{1}\right) \Gamma^{\prime}\left(\theta_{1}\right)=\Gamma\left(\theta_{2}\right) \cos \left(\theta_{2}\right)-\sin \left(\theta_{2}\right) \Gamma^{\prime}\left(\theta_{2}\right)
$$

Eliminating $\Gamma^{\prime}\left(\theta_{1}\right)$ and $\Gamma^{\prime}\left(\theta_{2}\right)$ between $(2.13),(2.14)$ and $(2.15)$ gives the identity

$$
\xi^{\star}=\frac{\Gamma\left(\theta_{1}\right)}{\cos \left(\theta_{1}\right)}=\frac{\Gamma\left(\theta_{2}\right)}{\cos \left(\theta_{2}\right)},
$$

where $\xi^{\star}=\left|\overrightarrow{\xi^{\star}}\right|$. This provides a relation between $\theta_{1}$ and $\theta_{2}$ as a result of the force balance at the edge.

\section{(b) Phase-field Model}

\section{(i) Isotropic Surface Energy}

We introduce a continuous phase-field function whose value denotes the state of the system at each point in space: $\phi=1$ is liquid, $\phi=0$ solid and intermediate 
values occur through interfaces. The dimensional Helmholtz free energy functional is assumed to be given by

$$
\mathcal{F}=\int \mathcal{L}(\phi, \nabla \phi) d V
$$

where the free energy density, $\mathcal{L}(\phi, \nabla \phi)$, is defined by

$$
\frac{1}{2} \hat{\eta}^{2}[\Gamma(\nabla \phi)]^{2}+\hat{f}(\phi, \hat{T}) .
$$

Here $\hat{\eta}$ is a constant, $\Gamma(\nabla \phi)$ is defined by $(2.4)$ and $\hat{f}(\phi, \hat{T})$ is the bulk free energy density. The governing equation is then given by a simple rate equation which ensures that Helmholtz free energy of the system decreases with time

$$
\frac{1}{\hat{m}(\nabla \phi)} \frac{\partial \phi}{\partial \hat{t}}=-\frac{\delta \mathcal{F}}{\delta \phi}
$$

where $\hat{m}(\nabla \phi)(>0)$ is a homogeneous degree one function that is related to the kinetic anisotropy of the interface. The dimensionless phase-field equation may then be expressed as

$$
\frac{\epsilon^{2}}{M} \frac{\Gamma(\nabla \phi)}{m(\nabla \phi)} \frac{\partial \phi}{\partial t}=\epsilon^{2} 72 \gamma^{2} \nabla \cdot[\Gamma(\nabla \phi) \vec{\xi}(\nabla \phi)]-G^{\prime}(\phi)+\epsilon H^{\prime}(\phi) u
$$

where $f(\phi, \hat{T})=G(\phi)-\epsilon H(\phi) u, G(\phi)=\phi^{2}(1-\phi)^{2} / 4, H(\phi)=\phi^{2}(3-2 \phi)$. The dimensionless constants can be determined by the physical properties of the interface by relating the one dimensional travelling wave solution of (2.20) to the physical properties of the interface, see Wheeler \& McFadden (1996):

$$
\gamma=\frac{\hat{\gamma}_{0}}{\hat{L} \hat{R}}, \quad \mu=\frac{\hat{\tau} \hat{T}_{M} \hat{\mu}_{0}}{\hat{R}}, \quad M=\frac{\mu}{72 \gamma}, \quad \epsilon=\frac{\hat{d} \hat{L}}{24 \hat{\gamma}_{0}},
$$

where a hat denotes a dimensional quantity. Here the surface energy is given by $\hat{\gamma}=\hat{\gamma}_{0} \Gamma(\vec{n})$, the kinetic parameter by $\hat{\mu}=\hat{\mu}_{0} m(\vec{n})$, where $\vec{n}$ is the outward unit normal to the interface, the interface thickness by $\hat{d}$, the macroscopic length scale of the interface by $\hat{R}$, the timescale by $\hat{\tau}$ and the latent heat per unit volume by $\hat{L}$. The precise choice of time scale is not important to the discussion, provided that the dimensionless kinetic coefficient, $M$, is order one. The dimensionless interfacial temperature is given by

$$
u=\frac{\hat{T}-\hat{T}_{M}}{\hat{T}_{M}}
$$

The parameter $\epsilon$ may be re-expressed as $\epsilon=1 /(24 \gamma)[\hat{d} / \hat{R}]$ and represents the ratio of the interface thickness to the macroscopic length scale and is therefore a small quantity. We observe from equation (2.20) that the length scale of the interface where $\phi$ varies between 0 and 1 scales with $\epsilon$, i.e, on the short length scale associated with the interface thickness. Thus we introduce a further rescaling of length so that the governing equation is appropriate to the description of the interfacial region. We also take the opportunity to scale time so that the resulting 
equation is independent of $\gamma$ and $M$. We put $\vec{x}=6 \sqrt{2} \epsilon \gamma \vec{x}_{\star}$ and $t=[1 / M] t_{\star}$, in which case the phase-field equation may be expressed as

$$
\epsilon^{2} \frac{\Gamma(\nabla \phi)}{m(\nabla \phi)} \frac{\partial \phi}{\partial t}=\nabla \cdot[\Gamma(\nabla \phi) \vec{\xi}(\nabla \phi)]-G^{\prime}(\phi)+\epsilon H^{\prime}(\phi) u,
$$

where the subscript stars have been omitted.

In $(2.21)$ the $\xi$-vector, $\vec{\xi}$, naturally appears being again given by the definition (2.5), but with an argument of $\nabla \phi$. This is the so-called generalised $\xi$-vector and, unlike in the sharp interface theory, it is defined throughout the whole domain and not confined to the surface of the interface, $\mathcal{I}$. It may be shown that providing the inverse $\gamma$-polar plot is convex the equation (2.21) is a forward parabolic partial differential equation. However, should it be non-convex then for those orientations of $\nabla \phi$ that correspond to non-convexity of the surface the equation locally becomes a backward parabolic partial differential equation. This corresponds to negative surface stiffness.

There is a one dimensional travelling wave solution of (2.21) which represents a planar interface moving with speed $V$ in a direction $\vec{n}$ is given by

$$
\begin{gathered}
\phi(x, t)=G_{0}\left(\frac{x-V t}{\Gamma(\vec{n})}\right), \\
G_{0}(r)=\frac{1}{2}\left[1+\tanh \left(\frac{r}{2 \sqrt{2}}\right)\right],
\end{gathered}
$$

when $u=-V /[\mu m(\vec{n})]$.

Using the above solution Wheeler and McFadden (1996) showed that in the sharp interface limit, $\epsilon \rightarrow 0$, in which the thickness of the interface, compared to the macroscopic length scale, goes to zero the anisotropic form of the GibbsThomson equation (2.3) is recovered. Wheeler and McFadden (1997) subsequently investigated the structure of edges. As has been noted above in the sharp interface theory, at an edge the $\xi$-vector is continuous, denoted here by $\vec{\xi}^{\star}$. They showed that in the phase-field theory edges are represented as weak shocks at a straight line aligned with the direction of $\vec{\xi}^{\star}$, across which the phase field is continuous but its gradient is not. They also showed that $\Gamma(\nabla \phi)$ is continuous across the shock. This, along with continuity of $\phi$, implies that the free energy density, $\mathcal{L}$, is also continuous across the shock.

Wheeler and McFadden also demonstrated that there existed a so-called $\xi$-tensor defined by

$$
\Xi=\gamma(\nabla \phi) \nabla \phi \otimes \vec{\xi}-\mathcal{L} I,
$$

which satisfies $\nabla \cdot \Xi=0$. Using this they were able to recover the classical force balance (2.10) across the edge.

\section{Diffuse Edge Theory}

\section{(a) Governing Equation}

As described above an edge in the phase-field theory is represented by a weak shock involving a discontinuity in $\nabla \phi$. Here we seek to regularise this discontinuity 
by introducing a generalised free energy of the form

$$
\mathcal{F}=\int\left[\frac{1}{2} \hat{\delta}^{2}[\Delta \phi]^{2}+\frac{1}{2} \hat{\eta}\left[\Gamma^{2}(\nabla \phi)\right]^{2}+\hat{f}(\phi, \hat{T})\right] d V,
$$

where we have extended the original functional associated with the phase-field model (2.17) by adding a term involving the square of the Laplacian of the phase field. Here $\hat{\delta}$ is a phenomenological constant. We will show that in this model an edge region is present through which the interfacial orientation continuously varies through the range of missing orientations, thus smoothing out the corner in $\mathcal{S}$. The parameter, $\hat{\delta}$, characterises the thickness of the edge region and is also related to the so-called 'edge energy' which is the excess free energy that, in this new model, is associated with the presence of the edge.

We assume that the dynamics are given by (2.19). Thus the dimensionless phasefield equation associated with this generalised model is an extension of (2.21). It is given by

$$
\epsilon^{2} \frac{\Gamma(\nabla \phi)}{m(\nabla \phi)} \frac{\partial \phi}{\partial t}=-\delta^{2} \Delta^{2} \phi+\nabla \cdot[\Gamma(\nabla \phi) \vec{\xi}]-G^{\prime}(\phi)+\epsilon H^{\prime}(\phi) u,
$$

where $\delta$ is a non-dimensional representation of the coefficient of the new square Laplacian contribution to the free energy density. It is related to the dimensional parameters by

$$
\delta=\hat{\delta} \frac{1}{432 \sqrt{2}} \frac{\hat{d}^{1 / 2} \hat{L}^{2}}{\hat{\gamma}^{5 / 2}} .
$$

\section{(b) Equilibrium Edge Structure: Limit $\epsilon \rightarrow 0, \delta \rightarrow 0$}

We now consider the internal structure of an isothermal edge, at equilibrium and at a temperature below the freezing temperature ( $u$ is a negative constant) in the limit, $\epsilon \rightarrow 0$, followed by the limit $\delta \rightarrow 0$. We consider the situation for a cylindrical interface. In the conventional sharp interface representation the edge resides at the corner formed from the intersection of the two adjoining interfaces represented as curved portions of the cross section $\mathcal{S}$. However, in the generalised diffuse edge model the interfaces and corner will all have a small thickness with a corresponding internal structure. We assume that the system is stationary and the interface regions meet in the inner edge region, at a contact angle given by the equilibrium value of the classical force balance at the edge. The detailed configuration in the vicinity of the edge is shown in Figure 4.

The edge has a five region structure: two outer regions representing the bulk phases, where $\phi$ is zero or unity; two intermediate regions representing the interfacial layers away from the edge in which $\phi$ makes a transition between its bulk values; the inner edge region which represents the confluence of the intermediate region and within which $\phi$ and $\nabla \phi$ vary continuously between their values in the four adjoining regions.

The two intermediate solutions will be denoted regions $I$ and $I I$, exterior to each side of the inner edge region itself. In each intermediate region we set up cartesian coordinate systems $\left(r_{I}, s_{I}\right)$ and $\left(r_{I I}, s_{I I}\right)$ which are aligned to the orientation of the interfaces. Given the equivalence of the two outer regions we will only discuss intermediate region $I$ in detail. In the inner edge region we establish another cartesian 
coordinate system $(r, s)$ which is aligned to the common value of the $\xi$-vector at the edge, $\vec{\xi}^{\star}$. We locate the common origin of the coordinate systems to correspond to $\phi=1 / 2$ in the inner edge region. Simple trigonometry gives the relation between the two coordinate systems as

$$
r_{I}=r \cos \theta_{1}+s \sin \theta_{1}, s_{I}=-r \sin \theta_{1}+s \cos \theta_{1},
$$

where $\theta_{1}$ represents the orientation of the interface as shown in figure 4 .

The dimensionless governing equation (3.2) is represented on the length scale associated with the intermediate regions. The inner edge region connects the two intermediate regions within which the gradient of the phase-field variable varies rapidly, rather than undergoing a discontinuity as in the diffuse interface model.

We now go on to consider the intermediate and inner edge regions in turn. We develop the solution in each region using the method of matched asymptotic expansion to match them together.

\section{(c) Intermediate Region I}

We first take the limit $\epsilon \rightarrow 0$. At leading order the governing equation (3.2) is

$$
-\delta^{2} \Delta^{2} \phi+\nabla \cdot[\Gamma(\nabla \phi) \vec{\xi}]-G^{\prime}(\phi)=0
$$

We develop its solution as a regular perturbation series in $\delta$. It is straight forward to show that this is given by

$$
\phi_{I}\left(r_{I}, s_{I}\right)=G_{0}\left(\frac{r_{I}}{\Gamma\left(\theta_{1}\right)}+B_{1} \delta\right)+\mathcal{O}\left(\delta^{2}\right),
$$

where $B_{1}$ is an undetermined constant which represents a translation of the interface.

Consideration of the development of the solution at first order in $\epsilon$ involves a similar secondary expansion as a regular perturbation series in $\delta$ as the leading order problem above. The solution of the leading order problem in this $\delta$ expansion at $\mathcal{O}(\epsilon)$ is identical to the analysis of Wheeler \& McFadden (1996) and we deduce that the temperature is related to the curvature of the interface by

$$
u=\gamma\left[\Gamma\left(\theta_{1}\right)+\Gamma_{\theta \theta}\left(\theta_{1}\right)\right] \mathcal{K}_{1}+\mathcal{O}(\delta),
$$

where $\mathcal{K}_{1}$ is the curvature of interface $I$ at the edge (non-dimensionalised with respect to $R$ ).

\section{(d) Inner Edge Region}

Again we first take the limit $\epsilon \rightarrow 0$. At leading order in $\epsilon$ we recover the governing equation (3.4) and consider its solution in the subsequent limit $\delta \rightarrow 0$. In this case we are unable to find a solution as a simple regular perturbation series, as was the case in the interfacial layers. Thus we seek a singular perturbation solution in which the biharmonic terms play a role at leading order and allow the inner edge region to support a continuous transition of the gradient of the phase field, and hence interface orientation, between its values in the interfacial regions, with 
interface orientations of $\theta_{1}$ and $\theta_{2}$. In order to accomplish this we introduce a thin inner edge region. To achieve an appropriate balance of terms in (3.4) where the biharmonic terms balance the interfacial gradient energy terms it is necessary to scale the lateral coordinate $s$ by $\delta$. We put

$$
s=\delta \sigma .
$$

The inner edge region is therefore of lateral thickness $\delta$. We will confine our attention to the solution of the leading order problem in $\epsilon$, given by (3.4), as $\delta \rightarrow 0$. We denote the phase field in the inner edge region by $\Phi(\sigma, r)$ and express it in the form

$$
\Phi(r, \sigma)=\Phi^{(0)}(\sigma, r)+\delta \Phi^{(1)}(\sigma, r)+\mathcal{O}\left(\delta^{2}\right)+\mathcal{O}(\epsilon) .
$$

The expansion of (3.4) in terms of the representation (3.7) is given in Appendix A.

The inner expansion, appropriate to the edge region, of the intermediate solution is found from expressing it in terms of the inner edge region coordinates $r$ and $\sigma$ and taking the limit $\delta \rightarrow 0$. This gives

$$
\phi \sim G_{0}\left(\frac{r \cos \theta_{1}}{\Gamma\left(\theta_{1}\right)}\right)+\delta G_{0}^{\prime}\left(\frac{r \cos \theta_{1}}{\Gamma\left(\theta_{1}\right)}\right)\left[\frac{\sigma \sin \theta_{1}}{\Gamma\left(\theta_{1}\right)}+B_{1}\right]+\mathcal{O}\left(\delta^{2}\right)+\mathcal{O}(\epsilon) .
$$

(e) Leading Order Edge Solution

The leading order problem, at $\mathcal{O}\left(\delta^{-2}\right)$, is

$$
\frac{\partial^{4} \Phi^{(0)}}{\partial \sigma^{4}}=0
$$

The matching condition is derived from the leading order representation of the intermediate solution given by (3.8). Thus

$$
\lim _{\sigma \rightarrow+\infty} \Phi^{(0)}(\sigma, r)=G_{0}\left(\frac{r \cos \theta_{1}}{\Gamma\left(\theta_{1}\right)}\right) \text { and } \lim _{\sigma \rightarrow-\infty} \Phi^{(0)}(\sigma, r)=G_{0}\left(\frac{r \cos \theta_{2}}{\Gamma\left(\theta_{2}\right)}\right) .
$$

We deduce that

$$
\Phi^{(0)}(\sigma, r)=G(r)
$$

where matching it with Intermediate Region $I$ requires

$$
G(r)=G_{0}\left(\frac{r \cos \theta_{1}}{\Gamma\left(\theta_{1}\right)}\right)
$$

Matching the phase-field with the Intermediate Region $I I$ requires that

$$
G_{0}\left(\frac{r \cos \theta_{1}}{\Gamma\left(\theta_{1}\right)}\right)=G_{0}\left(\frac{r \cos \theta_{2}}{\Gamma\left(\theta_{2}\right)}\right),
$$

and hence

$$
\frac{\cos \theta_{1}}{\Gamma\left(\theta_{1}\right)}=\frac{\cos \theta_{2}}{\Gamma\left(\theta_{2}\right)}
$$

However, this relationship is automatically satisfied as we have assumed the system is in equilibrium and therefore subject to the classical force balance at the edge which requires the same relation (2.16) to hold. We note that we may conveniently write equation (3.10) as

$$
G(r)=G_{0}\left(\frac{r}{\xi^{\star}}\right) .
$$




\section{(f) First Order Edge Solution}

From equation $(3.4)$ at $\mathcal{O}\left(\delta^{-1}\right)$

$$
-\frac{\partial^{4} \Phi^{(1)}}{\partial \sigma^{4}}+\frac{\partial}{\partial \sigma}\left[\Gamma\left(\nabla^{(0)} \Phi\right) \frac{\partial \Gamma\left(\nabla^{(0)} \Phi\right)}{\partial \Phi_{1, \sigma}^{(0)}}\right]=0,
$$

where

$$
\nabla^{(0)} \Phi=\frac{\partial \Phi^{(1)}}{\partial \sigma} \vec{M}+\frac{\partial \Phi^{(0)}}{\partial r} \vec{N}=\frac{\partial \Phi^{(1)}}{\partial \sigma} \vec{M}+G^{\prime}(r) \vec{N} .
$$

To simplify the notation we write

$$
u=\frac{\partial \Phi^{(1)}}{\partial \sigma},
$$

and hence (3.13) may be written as

$$
-\frac{\partial^{3} u}{\partial \sigma^{3}}+\frac{\partial}{\partial \sigma}\left[\Gamma\left(\nabla^{(0)} \Phi\right) \frac{\partial \Gamma\left(\nabla^{(0)} \Phi\right)}{\partial u}\right]=0,
$$

where

$$
\nabla^{(0)} \Phi=u \vec{M}+G^{\prime}(r) \vec{N} .
$$

This may be integrated once to give

$$
-\frac{\partial^{2} u}{\partial \sigma^{2}}+\Gamma\left(\nabla^{(0)} \Phi\right) \frac{\partial \Gamma\left(\nabla^{(0)} \Phi\right)}{\partial u}=\text { constant. }
$$

Now

$$
\frac{\partial \Gamma\left(\nabla^{(0)} \Phi\right)}{\partial u}=\vec{\xi} \cdot \vec{M} \rightarrow 0 \text { as } \sigma \rightarrow \pm \infty,
$$

as $\vec{\xi} \cdot \vec{M}=0$ in the interfacial layers. Thus the constant above is zero and we may integrate again to obtain

$$
-\frac{1}{2}\left[u_{\sigma}\right]^{2}+\frac{1}{2}\left[\Gamma\left(\nabla^{(0)} \Phi\right)\right]^{2}=\frac{1}{2} \Gamma_{\infty}^{2}
$$

and hence

$$
u_{\sigma}=\sqrt{\left[\Gamma\left(\nabla^{(0)} \Phi\right)\right]^{2}-\Gamma_{\infty}^{2}},
$$

where $\Gamma_{\infty}$ is the common value of $\Gamma(\nabla \phi)$ required by the matching across the edge region.

We define $\Theta$ as the angle that $\nabla^{(0)} \Phi$ makes with $\vec{N}$ which is given by

$$
u=\frac{\partial \Phi^{(1)}}{\partial \sigma}=G^{\prime}(r) \tan \Theta .
$$

Thus $u=G^{\prime}(r) \tan \Theta$ and hence $u_{\sigma}=\Theta_{\sigma} G^{\prime}(r) \sec ^{2} \Theta=\Theta_{\sigma}\left[u^{2}+\left(G^{\prime}(r)\right)^{2}\right] / G^{\prime}(r)$ and we find that

$$
\Theta_{\sigma}=\frac{G^{\prime}(r)}{\sqrt{u^{2}+\left(G^{\prime}(r)\right)^{2}}} \sqrt{[\Gamma(\Theta)]^{2}-\cos ^{2} \Theta\left[\frac{\Gamma\left(\theta_{1}\right)}{\cos \theta_{1}}\right]^{2}},
$$


where we have have used the property that $\Gamma(\nabla \phi)$ is a first order homogeneous function and written $\Gamma\left(\nabla \Phi^{(0)}\right)=\left|\nabla \Phi^{(0)}\right| \Gamma(\Theta)$. Hence

$$
\Theta_{\sigma}=\cos (\Theta) \sqrt{[\Gamma(\Theta)]^{2}-\cos ^{2} \Theta\left[\xi^{\star}\right]^{2}} .
$$

Integrating equation (3.21) with respect to $\sigma$ gives the first order correction to the phase field in the edge region as

$$
\Phi^{(1)}=G^{\prime}(r) \mathcal{H}(\sigma)+A(r),
$$

where $A(r)$ is a, as yet undetermined, function of $r$, and

$$
\mathcal{H}(\sigma)=\int_{0}^{\sigma} \tan \Theta d \sigma=i n t_{0}^{\Theta(\sigma)} \frac{\tan \Theta^{\prime}}{\cos \left(\Theta^{\prime}\right) \sqrt{\left[\Gamma\left(\Theta^{\prime}\right)\right]^{2}-\cos ^{2} \Theta^{\prime}\left[\xi^{\star}\right]^{2}}} d \Theta^{\prime} .
$$

To determine $A(r)$ we match the inner and intermediate solution to $\mathcal{O}(\delta)$. The intermediate expansion of $\Phi$ is given by

$$
\Phi \sim G_{0}\left(\frac{r \cos \theta_{1}}{\Gamma\left(\theta_{1}\right)}\right)+\delta \frac{\cos \theta_{1}}{\Gamma\left(\theta_{1}\right)} G_{0}^{\prime}\left(\frac{r \cos \theta_{1}}{\Gamma\left(\theta_{1}\right)}\right)\left[\sigma \tan \theta_{1}+T(\infty)+A(r)\right]+\mathcal{O}\left(\delta^{2}\right),
$$

We have re-expressed $\mathcal{H}$, given by (3.27), as

$$
\mathcal{H}(\sigma)=\sigma \tan \theta_{1}+\mathcal{T}(\sigma)
$$

for $\sigma>0$, where

$$
\mathcal{T}(\sigma)=\int_{0}^{\sigma}\left(\tan \Theta-\tan \theta_{1}\right) d \sigma
$$

A comparison of the $\mathcal{O}(\delta)$ terms in (3.8) and (3.26) shows that the terms proportional to $\sigma$ automatically match and that

$$
B_{1}=\frac{\cos \theta_{1}}{\Gamma\left(\theta_{1}\right)}[A(r)+\mathcal{T}(\infty)]
$$

We deduce that $A(r)$ is a constant. However, we have co-located the common origin of the coordinate systems with $\phi=1 / 2$ in the inner edge region and thus we require that $A(r)=0$ and

$$
B_{1}=\mathcal{T}(\infty) \frac{\cos \left(\theta_{1}\right)}{\Gamma\left(\theta_{1}\right)}=\frac{\mathcal{T}(\infty)}{\xi^{\star}}
$$

Thus the interfaces adjoining the edge are translated by an extent $\mathcal{O}(\delta)$ with respect to the edge itself.

From equations (3.9) and (3.24) the phase field in the inner edge region is given as

$$
\Phi(\sigma, r)=G(r+\delta \mathcal{H}(s / \delta))+\mathcal{O}\left(\delta^{2}\right)+\mathcal{O}(\epsilon)
$$

The contours of $\Phi$, and hence the shape of the interface in the inner edge region, are approximated by

$$
r+\delta \mathcal{H}(s / \delta)=\text { constant }
$$

We have integrated (3.23) and (3.27) numerically for the case $\Gamma(\theta)=1+$ $0.2 \cos (4 \theta)$ to determine $\Theta(\sigma)$ and $\mathcal{H}(\sigma)$ and hence the phase field in the inner edge region. In Figure 5 we show the interface shape given by the contour $\Phi(s, r)=1 / 2$ and in Figure 6 we plot $\Phi(s, r)$. 


\section{Edge Energy}

In this section we consider the excess energy associated with the edge in this generalised phase-field model. We define the edge 'edge energy', $\mathcal{F}_{X}$, in an analogous way to the surface energy in the phase-field model, i.e., the difference between the free energy of the system with and without the square Laplacian terms in the underlying free energy functional:

$\mathcal{F}_{X}=\int_{-\infty}^{\infty} \int_{-\infty}^{\infty}\left\{\frac{1}{2} \delta^{2}[\Delta \Phi]^{2}+\frac{1}{2} \Gamma(\nabla \Phi)^{2}+f(\Phi)\right\} d r d s-\int_{-\infty}^{\infty} \int_{-\infty}^{\infty}\left\{\frac{1}{2} \Gamma(\nabla \phi)^{2}+f(\phi)\right\} d r d s$

Using the asymptotic expressions for $\phi$ and $\Phi$ in the inner edge region, derived above, it can be shown that

$$
\begin{gathered}
\frac{1}{2} \delta^{2}(\Delta \Phi)^{2}=\frac{1}{2} G^{\prime}(r)^{2} \sec ^{4} \Theta\left[\Theta_{\sigma}\right]^{2}+\mathcal{O}(\delta), \\
\Gamma(\nabla \Phi)^{2}-\Gamma(\nabla \phi)^{2}=G^{\prime}(r)^{2}\left[\sec ^{2} \Theta \Gamma(\Theta)^{2}-\left(\xi^{\star}\right)^{2}\right]+\mathcal{O}(\delta)
\end{gathered}
$$

and

$$
f(\Phi)-f(\phi)=\delta f_{\phi}(G(\rho)) G^{\prime}(\rho)\left[\mathcal{H}(\sigma)-\sigma \tan \Theta-B_{1}\right]+\mathcal{O}(\delta)^{2} .
$$

Inserting these forms into (4.1) gives that

$$
\mathcal{F}_{X}=\delta \int_{-\infty}^{\infty} G^{\prime}(r)^{2} d r \times \int_{-\infty}^{\infty}\left\{\frac{\Gamma(\Theta)^{2}}{\cos ^{2} \Theta}-\left(\xi^{\star}\right)^{2}\right\} d \sigma+\mathcal{O}\left(\delta^{2}\right)
$$

The first integral evaluates to $1 / \xi^{\star}$ and so the edge energy, at leading order, is given by

$$
\mathcal{F}_{X}=\frac{\delta}{\xi^{\star}} \int_{-\infty}^{\infty}\left\{\frac{\Gamma(\Theta)^{2}}{\cos ^{2} \Theta}-\left(\xi^{\star}\right)^{2}\right\} d \sigma .
$$

We may express this integral in terms of a quadrature with respect to $\Theta$ as

$$
\mathcal{F}_{X}=\delta \int_{-\theta_{2}}^{\theta_{1}} \sec ^{2} \Theta \sqrt{\frac{\Gamma(\Theta)^{2}}{\left(\xi^{\star}\right)^{2} \cos ^{2} \Theta}-1} d \Theta .
$$

For the case $\Gamma(\theta)=1+\alpha \cos (4 \theta)$ we have computed the edge energy, $\mathcal{F}_{X}$, as a function of $\alpha$ which represents the strength of the anisotropy. For this four fold symmetry an edge is present only when the surface stiffness is negative, which corresponds to $\alpha>1 / 15$.

\section{The $\Xi$-tensor}

In this section we show that there is a $\Xi$-tensor for the time independent eneralised phase-field model. The corresponding Euler-Lagrange equation is

$$
\Delta\left(\frac{\partial \mathcal{L}}{\partial \Delta \phi}\right)-\nabla \cdot\left(\frac{\partial \mathcal{L}}{\partial \nabla \phi}\right)+\frac{\partial \mathcal{L}}{\partial \phi}=0 .
$$

We consider

$$
\frac{d \mathcal{L}}{d x_{k}}=\frac{\partial \mathcal{L}}{\partial \Delta \phi} \frac{\partial \Delta \phi}{\partial x_{k}}+\frac{\partial \mathcal{L}}{\partial \phi_{, j}} \phi_{, j k}+\frac{\partial \mathcal{L}}{\partial \phi} \phi_{, k}+\frac{\partial \mathcal{L}}{\partial x_{k}}
$$


which, using the Euler-Lagrange equation (5.1), may be expressed as

$$
\begin{aligned}
\frac{d \mathcal{L}}{d x_{k}} & \left.=\frac{\partial \mathcal{L}}{\partial \Delta \phi} \frac{\partial \Delta \phi}{\partial x_{k}}+\frac{\partial \mathcal{L}}{\partial \phi, j} \phi_{, j k}+\phi_{, k}\left\{\frac{\partial}{\partial x_{j}}\left(\frac{\partial \mathcal{L}}{\partial \phi_{, j}}\right)-\frac{\partial^{2}}{\partial x_{j} \partial x_{j}}\left(\frac{\partial \mathcal{L}}{\partial \Delta \phi}\right)\right\}+\frac{\partial \mathcal{L}}{\partial \dot{f}_{k}}\right) \\
& =\frac{\partial \mathcal{L}}{\partial \Delta \phi} \nabla_{k} \Delta \phi-\Delta\left(\frac{\partial \mathcal{L}}{\partial \Delta \phi}\right) \nabla_{k} \phi+\frac{\partial \mathcal{L}}{\partial \phi_{, j}} \phi_{, j k}+\phi_{, k} \frac{\partial}{\partial x_{j}}\left(\frac{\partial \mathcal{L}}{\partial \phi_{, j}}\right) \\
& =\frac{\partial \mathcal{L}}{\partial \Delta \phi} \nabla_{k} \Delta \phi-\Delta\left(\frac{\partial \mathcal{L}}{\partial \Delta \phi}\right) \nabla_{k} \phi+\frac{d}{d x_{j}}\left[\phi_{, k} \frac{\partial \mathcal{L}}{\partial \phi_{, j}}\right]+\frac{\partial \mathcal{L}}{\partial x_{k}}
\end{aligned}
$$

Using the identity

$$
\nabla \cdot(\vec{\beta} \otimes \nabla \alpha)-\nabla \cdot(\alpha \nabla \vec{\beta})=\vec{\beta} \Delta \alpha-\alpha \Delta \vec{\beta}
$$

equation (5.5) may be expressed as

$$
\frac{d \mathcal{L}}{d x_{k}}-\frac{\partial \mathcal{L}}{\partial x_{k}}-\nabla \cdot\left(\nabla \phi \otimes \frac{\partial \mathcal{L}}{\partial \nabla \phi}\right)=\nabla \cdot\left(\nabla \phi \otimes \nabla\left(\frac{\partial \mathcal{L}}{\partial \Delta \phi}\right)-\frac{\partial \mathcal{L}}{\Delta \phi} \nabla \nabla \phi\right)
$$

As $\mathcal{L}$ is independent of position we can write this as the divergence of a tensor $\Xi$ :

$$
\nabla \cdot \boldsymbol{\Xi}=0
$$

where

$$
\boldsymbol{\Xi}=\mathcal{L} I-\nabla \phi \otimes \frac{\partial \mathcal{L}}{\partial \nabla \phi}+\nabla \phi \otimes \nabla \frac{\partial \mathcal{L}}{\partial \Delta \phi}-\frac{\partial \mathcal{L}}{\partial \Delta \phi} \nabla \nabla \phi
$$

where $I$ is the unit tensor. Evaluating the various terms gives the following (dimensiomless) form for the $\Xi$ tensor:

$$
\boldsymbol{\Xi}=\left[f(\phi, u)+\frac{1}{2} \Gamma^{2}(\nabla \phi)\right] I-\Gamma(\nabla \phi) \nabla \phi \otimes \vec{\xi}+\delta^{2}\{\nabla \phi \otimes \nabla(\Delta \phi)-\Delta \phi \nabla \nabla \phi\} .
$$

The last terms with coefficient $\delta^{2}$ represent the new contributions that are specific to the generalised phase-field model.

\section{Discussion}

In this paper we have investigated the internal structure of edges in a generalised phase-field model. It can be shown that the leading equation in the inner edge region governing the orientation of the interface (3.17) may be expressed as

$$
\frac{d^{2} q}{d s^{2}}=\frac{\partial[F(q)]^{2}}{\partial q}
$$

where $q=\tan \theta$ and $F(q)=\Gamma(\theta) / \cos \theta$. This is an Allen-Cahn equation. The potential $F(q)$ has a double-well form when the surface stiffness is negative for some orientations, i.e., corresponding to the formation of edges. Thus we may interpret the leading order inner edge solution as satisfying a common tangent construction on $\left(F(q)\right.$, spanning the values of $q$ at the minima of each well, denoted $q_{1}$ and $q_{2}$. 
The edge energy can be expressed as

$$
\mathcal{F}_{X}=\frac{\delta}{F\left(q_{1}\right)} \int_{-\infty}^{\infty}\left\{[F(q)]^{2}-\left[F\left(q_{1}\right)\right]^{2}\right\} d \sigma,
$$

and is therefore proportional to the area bounded by the common tangent construction and the double-well in $[F(q)]^{2}$ as well as the thickness of the edge region.

The common tangent construction on $F(q)$ was established by Cabrera (1964) who made the analogy with a phase transition. The theory presented here provides a diffuse edge version of Cabrera's ideas, by developing a governing equation for the interface orientation that satisfies the same common tangent construction. Thus the generalised phase-field model (3.2) contains within it a diffuse description of two types of phase transition involving $\phi$ and $\nabla \phi$ :

- The solid-liquid phase transition across a planar interface in which the value of $\phi$ varies continuously and automatically satisfies a common tangent construction on the bulk free energy function $f(\phi, T)$.

- The edge phase transition in which the orientation of $\nabla \phi$, and hence the interface, represented by $q$, automatically satisfies a common tangent construction on $F(q)$.

We have developed a model based on a diffuse interface description of an interface and have shown that it describes smoothed edges, albeit in the limit $\delta \rightarrow 0$. We can reasonably expect that smooth edges will also be present when $\delta$ is an order one quantity. In this case the thicknesses of the edge region and the adjoining interfaces would by comparable and this regime may well be useful in the numerical phase-field simulations in which edges form.

In this work we have not included the effect of stress. However, we have derived a 'stress tensor' which is a natural generalisation of that for the standard phasefield model. In that situation it may be shown that $\Xi$ constitutes the reversible part of the stress tensor in a treatment that allows for motion of the two phases Anderson et al (1998). This requires further work but may suggest that the effect of the regularisation may affect the force balance and hence the equilibrium angle between the adjoining interfaces at an edge once bulk stress effects are explicitly included.

\section{Summary}

To date investigations of corners and edges in interfaces in the presence of surface anisotropy have focussed on the regularisation of sharp interface models. In this paper we have considered this from the different perspective of a diffuse interface description of the interface. Specifically, we have extended the anisotropic phase-field equation, based on a generalised $\xi$-vector, by introducing a natural regularisation. This model provides a diffuse description of edges as well as interfaces. We considered the model in the singular limit, $\delta \rightarrow 0$, and were able to determine the interior structure of the edge region in which the edge is smoothed out and the interface orientations transition through the span of missing orientations associated with the edge in the Wullf shape. We are able to develop the analogy of the edge region as representing a phase transition first identified by Cabrera. 
We have determined the associated edge energy and shown that it scales with the regularisation coefficient, $\delta$. Lastly, we have derived a stress tensor associated with the generalised phase-field equation.

\section{Acknowledgement}

The author is very grateful to Dr G. B. McFadden for stimulating conversations during the conduct of this work.

\section{Appendix A. Representation of the Governing Equation in the Inner Edge Region}

Here we use the inner edge representation of the phase field given by (3.7 to expand the governing equation (3.4) (at leading order in $\epsilon$ ). We consider each operator in the equation in turn:

The biharmonic operator is written as

$$
\delta^{2} \Delta^{2} \Phi=\delta^{2}\left(\frac{\partial^{2}}{\partial r^{2}}+\frac{1}{\delta^{2}} \frac{\partial^{2}}{\partial \sigma^{2}}\right)^{2} \Phi=\frac{1}{\delta^{2}} \frac{\partial^{4} \Phi^{(0)}}{\partial \sigma^{4}}+\frac{1}{\delta}\left(\frac{\partial^{4} \Phi^{(1)}}{\partial \sigma^{4}}+2 \frac{\partial^{4} \Phi^{(0)}}{\partial \sigma^{2} \partial r^{2}}\right)+\mathcal{O}(1)
$$

The $\xi$-vector is given by

$$
\begin{aligned}
\vec{\xi} & =\frac{\partial \Gamma(\nabla \Phi)}{\partial \phi_{, s}} \vec{M}+\frac{\partial \Gamma(\nabla \Phi)}{\partial \phi_{, r}} \vec{N} \\
& =\delta \frac{\partial \Gamma(\nabla \Phi)}{\partial \phi_{, \sigma}} \vec{M}+\frac{\partial \Gamma(\nabla \Phi)}{\partial \phi_{, r}} \vec{N} \\
& =\frac{\partial \Gamma(\nabla \Phi)}{\partial \Phi_{1, \sigma}^{(0)}} \vec{M}+\frac{\partial \Gamma(\nabla \Phi)}{\partial \Phi_{0, r}^{(0)}} \vec{N}
\end{aligned}
$$

and

$$
\begin{aligned}
\nabla \Phi & =\frac{\partial \Phi}{\partial s} \vec{M}+\frac{\partial \Phi}{\partial r} \vec{N}(\delta) \\
& =\frac{\partial \Phi_{1}^{(0)}}{\partial \sigma} \vec{M}+\frac{\partial \Phi_{0}^{(0)}}{\partial r} \vec{N}+\mathcal{O}(\delta)
\end{aligned}
$$

Thus

$$
\nabla \cdot(\Gamma(\nabla \phi) \vec{\xi})=\frac{1}{\delta} \frac{\partial}{\partial \sigma}\left[\Gamma(\nabla \Phi) \frac{\partial \Gamma(\nabla \Phi)}{\partial \Phi_{1, \sigma}^{(0)}}\right]+\mathcal{O}(1)
$$

Hence the governing equation is expressed as

$$
-\frac{1}{\delta^{2}}\left(\frac{\partial^{4} \Phi^{(0)}}{\partial \sigma^{4}}\right)+\frac{1}{\delta}\left(-\frac{\partial^{4} \Phi^{(1)}}{\partial \sigma^{4}}-2 \frac{\partial^{4} \Phi^{(0)}}{\partial \sigma^{2} \partial r^{2}}+\frac{\partial}{\partial \sigma}\left[\Gamma\left(\nabla^{(0)} \Phi\right) \frac{\partial \Gamma\left(\nabla^{(0)} \Phi\right)}{\partial \Phi_{1, \sigma}^{(0)}}\right]\right)+\mathcal{O}(1)=0,
$$

where

$$
\nabla^{(0)} \Phi=\frac{\partial \Phi_{1}^{(0)}}{\partial \sigma} \vec{M}+G^{\prime}(r) \vec{N}
$$




\section{References}

Anderson, D.M., McFadden, G.B. \& Wheeler, A.A. 1998 Diffuse-interface methods in fluid mechanics. Annual Review of Fluid Mechanics, 30, 139-165.

Andreev, A. F. 1981 Sov. Phys JETP, 53, 1063.

Burton, W. K., Cabrera, N. \& Frank, F. C. 1951 The growth of crystals and the equilibrium structure of their surfaces, Phil. Trans. Roy. Soc. London, Series A, 243, 299-358.

Cabrera, N. 1963 in Symposium on Properties of Solid Surfaces (American Society for Testing and Materials, Philadelphia).

Caginalp, G. 1985 Surface tension and supercooling in solidification theory. In Applications of Field Theory to Statistical Mechanics, ed. L Garrido, pp. 216-26. Berlin: SpringerVerlag.

Caginalp G. 1986. An analysis of a phase field model of a free boundary. Arch. Rat. Mech. Anal. 92, 205-245.

Cahn, J. W. \& Hoffmann, D. W. 1974 A vector thermodynamics for anisotropic surfaces - II Curved and facetted surfaces Acta Metall. 22, 1205-1214.

Curie, P. 1885 Bull. Min. France, 8, 145.

Di Carlo, A., Gurtin, M. E., Podio-Guidugli, P. 1992 A regularized equation for anisotropic motion-by-curvature. SIAM J. Appl. Math, 52, 1111-1119.

Frank, F. C. 1963 Metal Surfaces (American Society for Metals, Metals Park, Ohio, USA.)

Gibbs, J. W. 1878 Trans. Conn. Acad., 3, 343.

Golovin, A. A., Davis, S. H., \& Nepomnyashchy, A. A. 1998 A convective Cahn-Hilliard model for the formation of facets and corners in crystal growth Physica D . 122, 202230 .

Golovin, A. A., Davis, S. H., \& Nepomnyashchy, A. A. 1999 Modeling the formation of facets and corners using a convective CahnHilliard model J. Cryst. Growth . 199, $1245-1250$.

Golovin A. A., Davis, S. H., \& Nepomnyashchy, A. A. 1999 Model for faceting in a kinetically controlled crystal growth. Phys. Rev. E, 59, 803-825.

Golovin, A. A., Nepomnyashchy, A. A. , Davis, S. H., \& Zaks, M. A. 2001 Convective Cahn-Hilliard models: From coarsening to roughening Phys. Rev. Lett. 86, 1550-1-553.

Hoffmannn, D. W. \& Cahn, J. W. 1972 A vector thermodynamics for anisotropic surfaces - I Fundamentals and applications to place surface junctions, Surface Science 31, 368388.

Herring, C. 1951 Some thereoms on the free energies of crystal surfaces. Physical Review, 82, 87-93.

Karma, A 1994 Phase field model of eutetctic growth. Phys. Rev. E, 39, 2245-2250.

Karma, A. \& Rappel, W. 1997 Phase-field simulation of three-dimensional dendrites: is microscopic solvability theory correct? J. Cryst. Growth 174, 54-64.

Kobayashi, R. 1992 Simulations of three dimensional dendrites. In Pattern Formation in Complex Dissipative Systems ed. Kai, S., World Scintific, Singapore, 121-128.

Langer, J. S. 1986 Models of pattern formation in first-order phase transitions. In it Directions in Condensed Matter Physics , ed. G Grinstein, G Mazenko, Philadelphia: World Sci. 165-186.

Liu, F. \& Metiu, H. 1993 Dynamics of phase separation of crystal surfaces. Phys. Rev. B, 48, 5808-5818.

Lord Rayleigh 1892 On the theory of surface forces II. Compressible fluids. Phil. Mag. 33 209-220.

Nestler, B. \& Wheeler, A. A. 1998 Anisotropic multi-phase-field model: Interfaces and junctions. Physical Review E, 57, 2602-2609.

Rowlinson, J. S. \& Widon, B. 1989. Molecular Theory of Capillarity Oxford Clarendon. 
Savina, T. V., Golovin, A. A., Davis, S. H., Nepomnyashchy, A. A. \& Voorhees, P. W. 2003 Faceting of a growing crystal surface by diffusion. Phys. Rev. E, 67, 021606-1-21606-16.

Spencer, B. J., 2004 Asymptotic solutions for the equilibrium crystal spape with small cormer energy regularisation. Phys. Rev. E. 69, 011603-1-011603-9

Stewart, J. \& Goldenfeld, N. 1992 Spinodal decompostion of a crystal surface Phys. Rev. $A$, 46, 6505-6512.

Taylor, J. E., J. W. Cahn, \& Handwerker, C. A. 1992 Geometric models of crystal growth. Acta Metall. Mater. 22, 1205-1214.

van der Waals J D 1893 The thermodynamic theory of capillarity under the hypothesis of a continuous density variation. Transl. JS Rowlinson, 1979, J. Stat. Phys. 20 197-244.

Warren, J. A., Murray B. T. 1996. Ostwald ripening and coalescence of a binary alloy in two dimensions using a phase-field model. Mod. Simul. Mater. Sci. Eng., 4, 215-229.

Wheeler, A. A., Murray, B. T. \& Schaeffer, R. J. 1993 Computation of dendrites using a phase-fiedl model. Physica D 66, 243-262.

Wheeler, A. A. \& McFadden, G. B. 1996 A $\xi$-vector formulation of anisotropic phase-field models: 3D asymptiotics. Euro. Jnl of Applied Mathematics, 7, 367-381.

Wheeler, A. A., McFadden \& Boettinger, W. J. 1996 Phase-field model for solidification of an eutectic alloy Proc. Roy. Soc. London A, 452 495-525.

Wheeler, A. A. \& McFadden, G. B. 1997 On the notion of a ?-vector and a stress tensor for a general class of anisotropic diffuse interface models. Proc. Roy. Soc. London, A, 453 1611-1630.

Wheeler, A. A. 1999 CahnHoffman $\xi$-Vector and its relation to diffuse interface models of phase transitions. Journal of Statistical Physics, 95, 1245-1280.

Wulff, G. 1901 Kristallogr. 34, 449. 


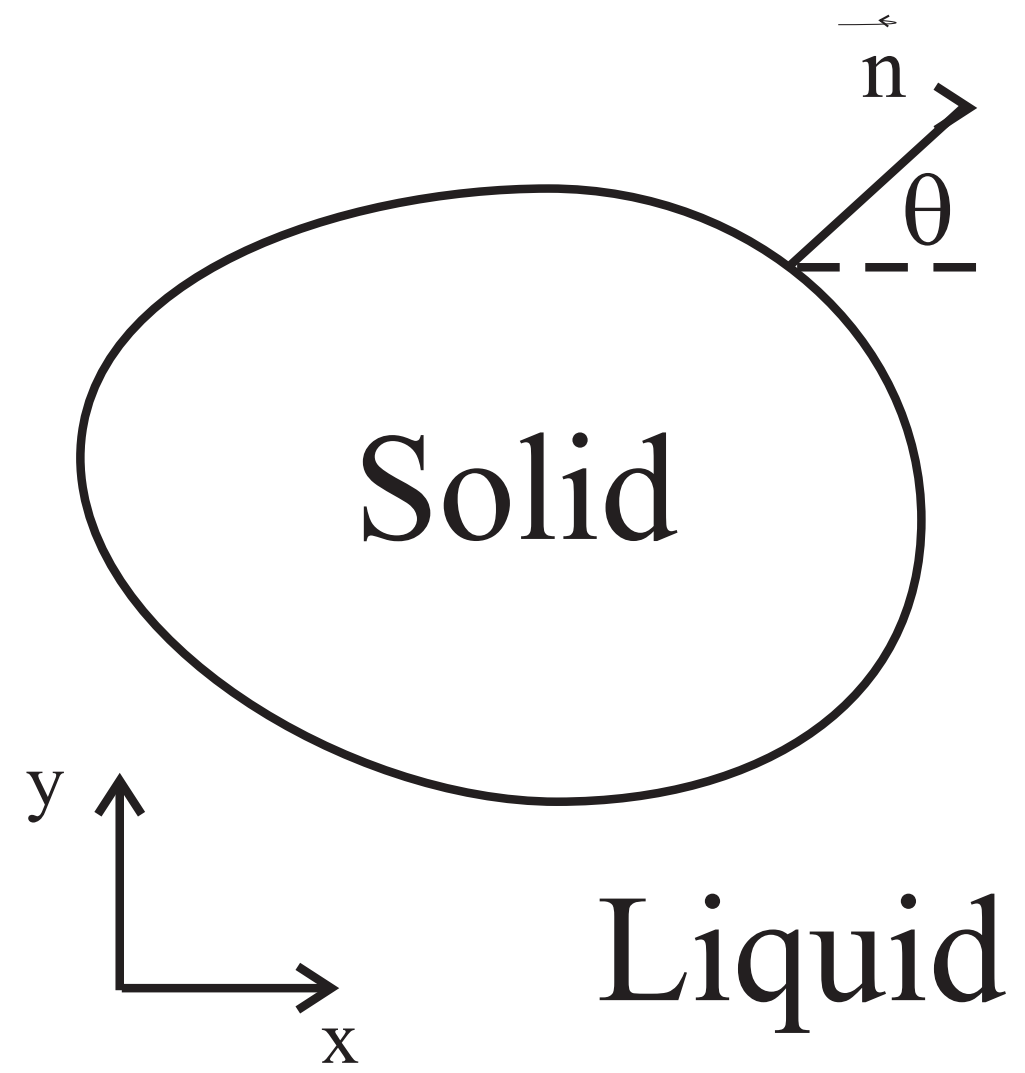

Figure 1. Configuration diagram of the general sharp interface situation. 


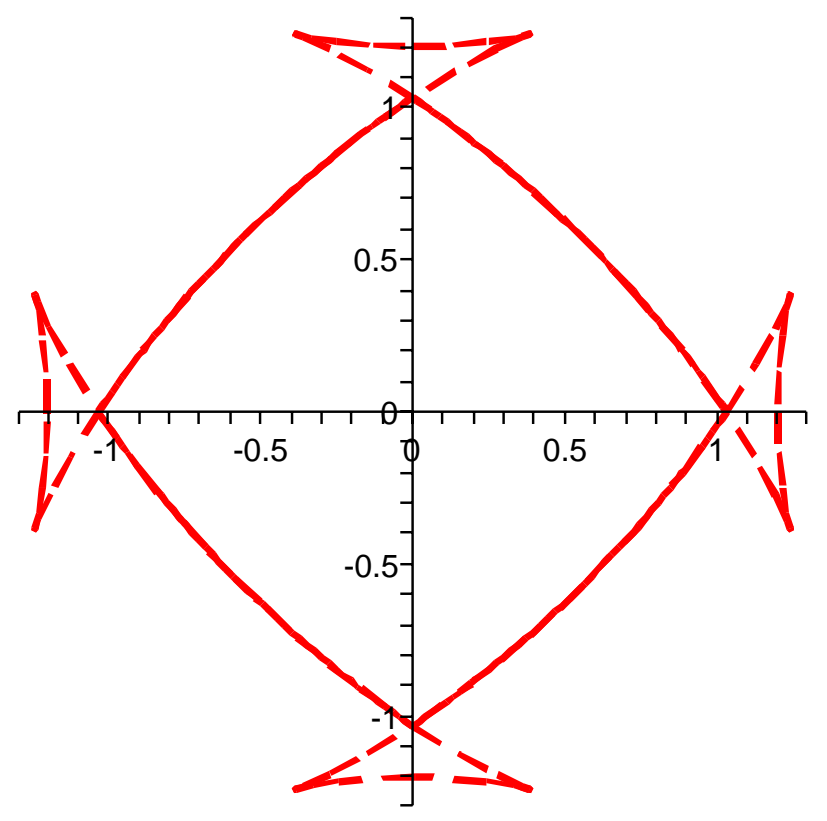

Figure 2. The shape of the interface computed using the $\xi$-vector for a model four-fold anisotropy given by $\Gamma)(\theta)=1+0.2 \cos (4 \theta)$. The solid curve represents the equilibrium shape of the solid, the dashed wings are parts of the $\xi$-vector plot corresponding to missing orientations.

Article submitted to Royal Society 


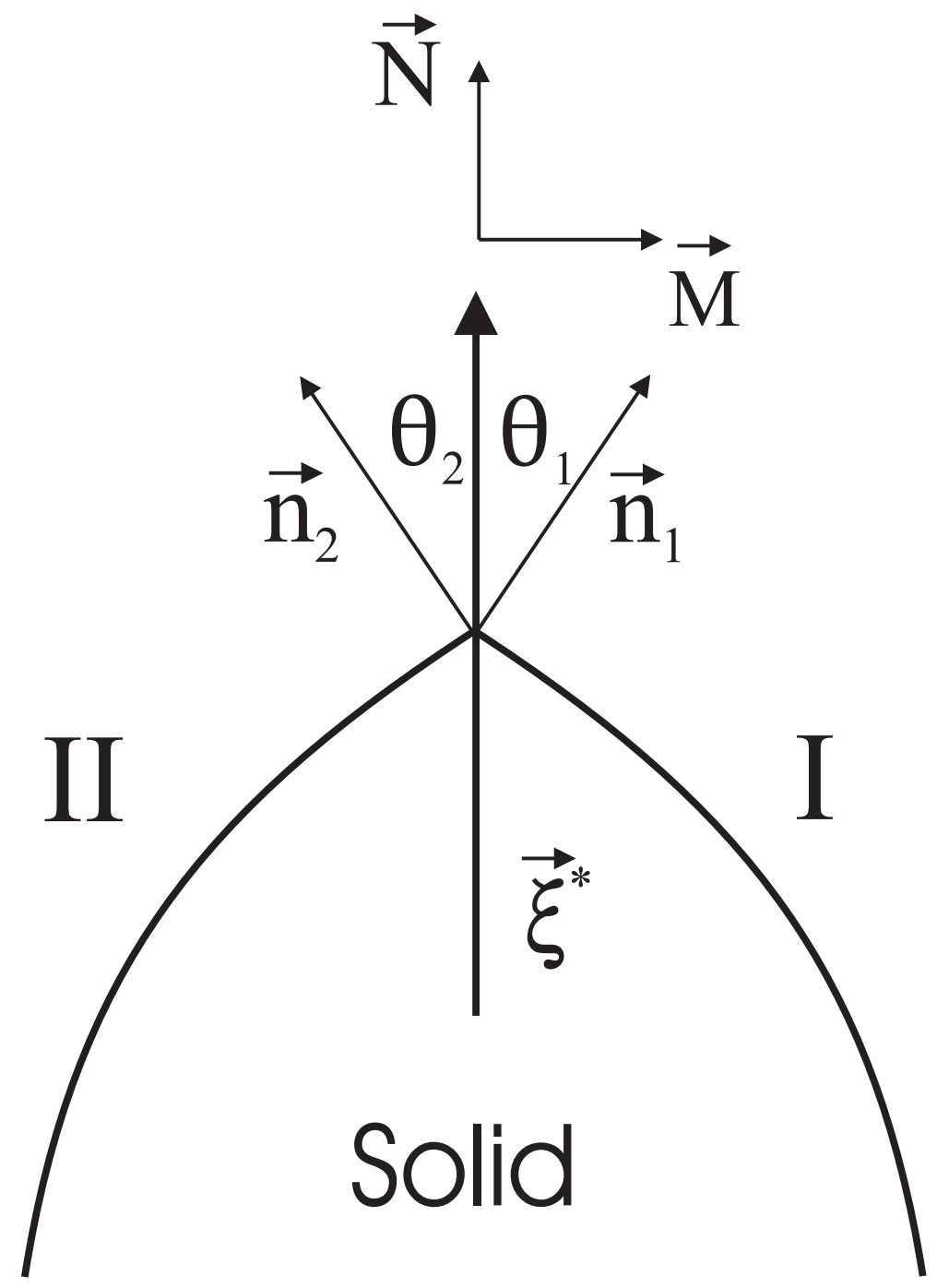

Figure 3. Configuration of the interface near an edge. 


\section{Liquid}

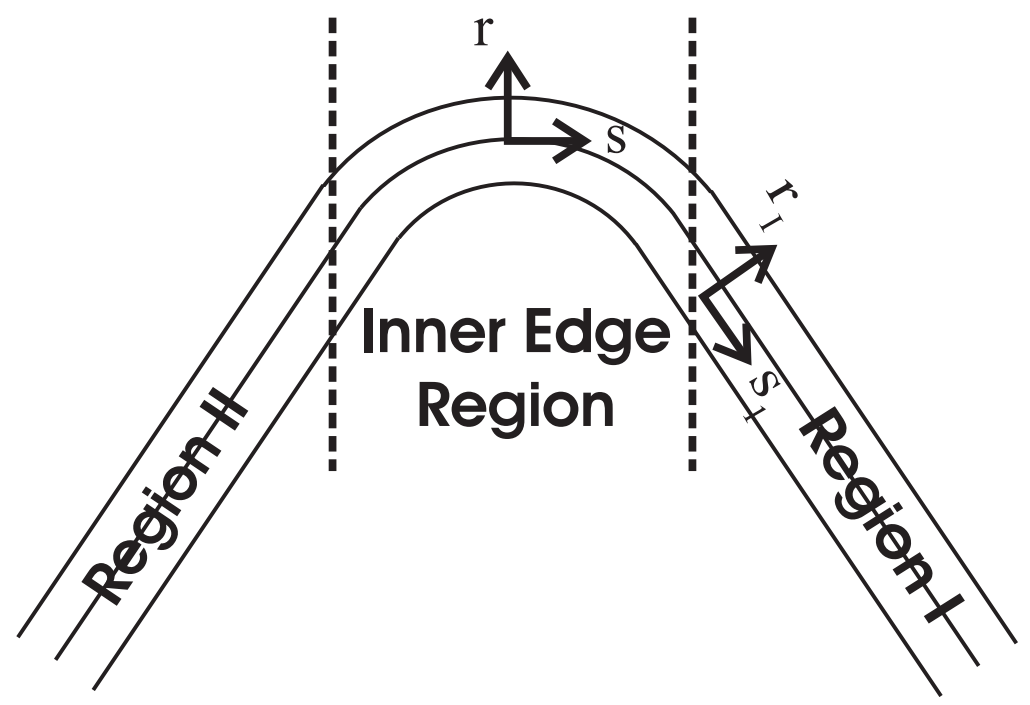

Solid

Figure 4. The relationship of the different regions. 


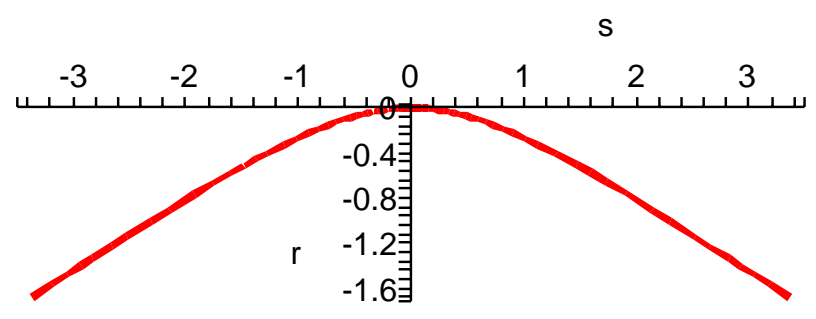

Figure 5. The shape of the interface in the inner edge region, as defined by the contour $\Phi(r, s)=1 / 2$, for model four-fold anisotropy given by $\Gamma(\theta)=1+0.2 \cos (4 \theta)$. 


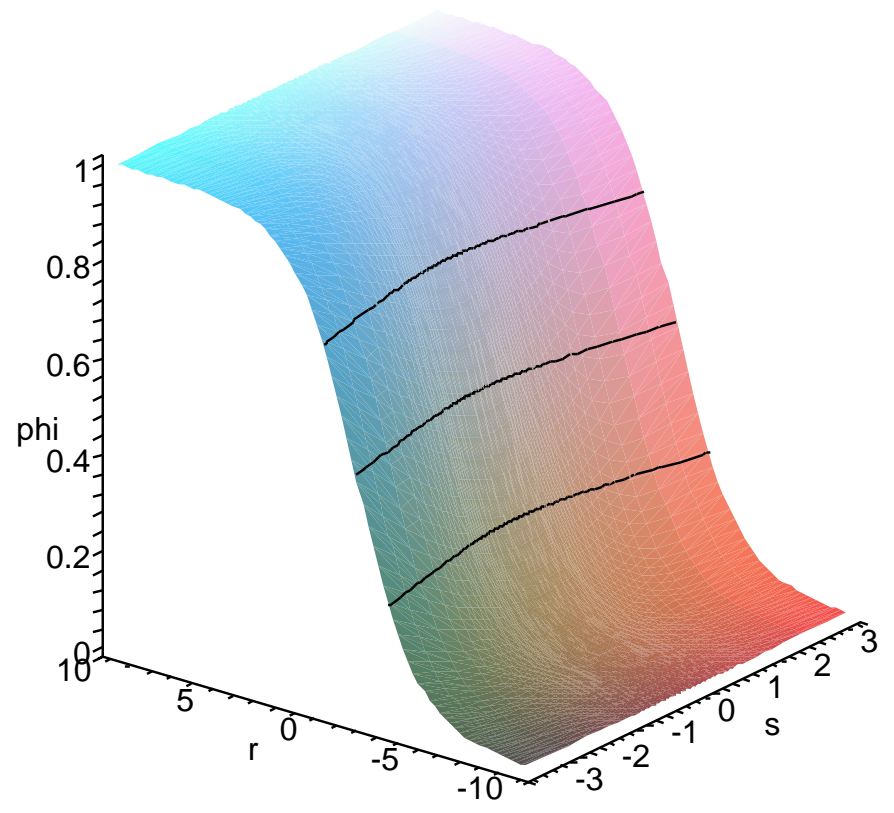

Figure 6 . The phase field in the inner region given by $\Phi(r, s)$, for the model four-fold anisotropy given by $\Gamma(\theta)=1+0.2 \cos (4 \theta)$. The solid black lines correspond to the contours $\phi=0.24,0.5,0.75$. 


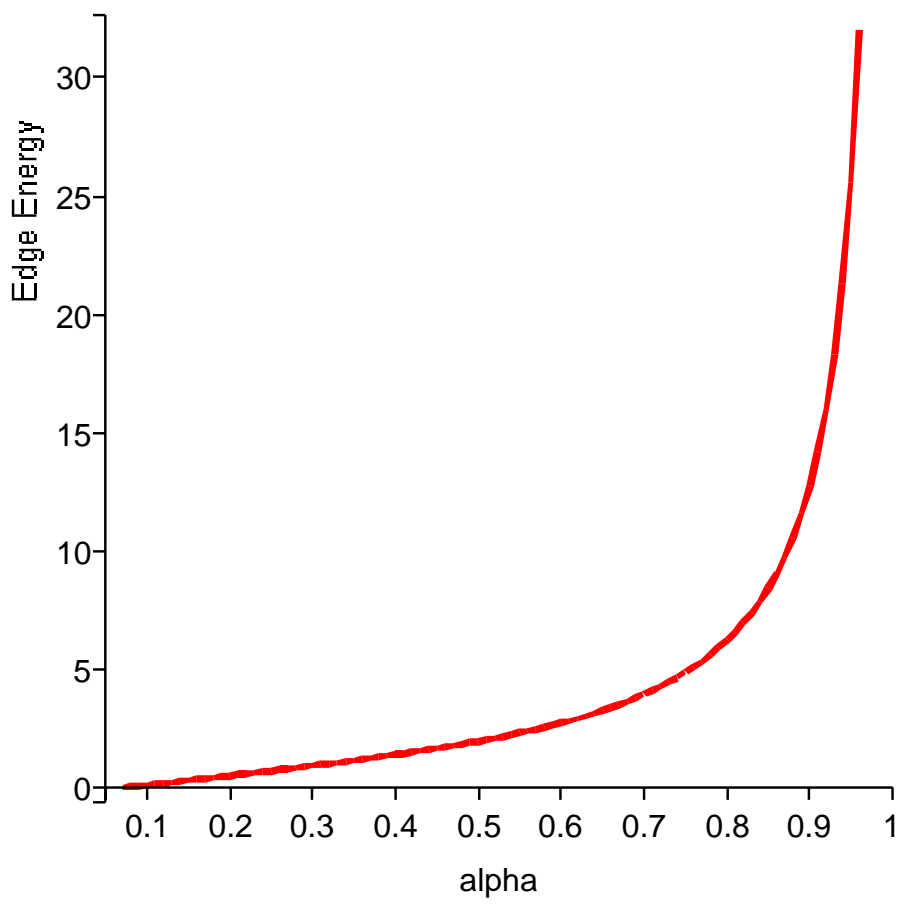

Figure 7. The dimensionless edge energy, $\mathcal{F}_{X}$, for the model four-fold anisotropy given by $\Gamma(\theta)=1+\alpha \cos (4 \theta)$ as a function of $\alpha$. The parameter $\alpha$ represents the strength of the anisotropy and an edge is present in the equilibrium shape for $\alpha>1 / 15$

Article submitted to Royal Society 